\title{
Schatten über den Anfängen ?
}

Wie viel sagen frühislamische Quellen über das aus, was wirklich war?

Von David Kiltz

\section{Einleitung}

\section{Quellenlage}

Die Quellenlage zur Entstehung und Anfangszeit des Islam ist bekannter- und anerkanntermaßen schwierig. Diese Anfangszeit beginnt mit dem traditionell für die Prophetentätigkeit Muhammads angenommene Periode von 610 bis 632 nach der Zeitenwende und dauert bis zum Ende der umayyadischen Herrschaft im Orient 750. Dem Historiker stehen hier im Wesentlichen drei Quellentypen zur Verfügung:

1. Die innerislamischen literarischen Quellen,

2. die außerislamischen literarischen Quellen und

3. Realien beziehungsweise Inschriften und bildliche Darstellungen, wobei bei letzteren Münzen eine besondere Bedeutung zukommt.

$\mathrm{Zu}$ den bedeutendsten innerislamischen literarischen Quellen gehört Ibn Ishâqs Sîrat rasûl allâh in den Überlieferungen von Ibn Hishâm und al-Tabarî sowie Yûnus Ibn Bukayr, welche etwa 150 Jahre nach dem Tod Muhammads verschriftlicht wurde. Diese Autoren berufen sich auf ältere Quellen wie etwa 'Urwa Ibn al-Zubayr Ibn al-'Awwâm, der etwa 635 geboren wurde und somit relativ nah an den Ereignissen zur Zeit des Propheten ist (Görke/Schoeler 2008). Die wichtigsten außerislamischen literarischen Quellen wurden 1997 von Robert G. Hoyland in Seeing Islam As Others Saw It. A Survey and Evaluation of Christian, Jewish and Zoroastrian Writings on Early Islam gesammelt. Das Werk beinhaltet griechische, lateinische, syrische, armenische, koptische, armenische und chinesische Quellen. Darunter finden sich Texte von Anastasius Sinaita, Thomas von Marga, Thomas dem Presbyter, Willibald von Eichstätt oder die historische Enzyklopädie Tongdian aus der Zeit der Tang-Dynastie in China. Die ältesten Quellen sind zeitgleich mit den letzten Lebensjahren Muhammads oder kurz nach seinem Tod entstanden. Münzen und einige Inschriften aus der Frühzeit des Islam liegen dem Historiker ebenfalls in geringer Menge vor.

All diese Quellen haben ihren spezifischen Nutzen und - nicht weniger wichtig - ihre Schwierigkeiten. In der Tat bedingt oft gerade die Bewertung dieser Quellen die vielleicht größten Unterschiede zwischen den verschiedenen Vorstellungen zur islamischen Frühzeit. Die hauptsächlichsten Interpretationsrichtungen sollen im Folgenden kurz skizziert werden.

\section{Ansätze}

\section{1. Übersicht}

Etwas vereinfacht, lässt sich von drei Hauptansätzen sprechen:

1. traditionalistisch

2. revisionistisch

3. integrativ

Die ,Traditionalisten ' in der islamischen Welt folgen im Wesentlichen der innerislamischen Überlieferung, das heißt der Tradition, welche als weitgehend historisch korrekt angesehen wird. Es sei hier allerdings erwähnt, dass es natürlich auch innerhalb der islamischen Welt häufig im Detail - vielfältige Unterschiede darin gibt, auf welche Aspekte der Überlieferung das zu beziehen ist. In der traditionellen westlichen Islamwissenschaft werden die klassischen islamischen Werke nach kritischer Sichtung ebenfalls als wesentliche Basis für die frühislamische Geschichte verwendet. Daneben finden sich so genannte ,revisionistische' 
Ansätze. Was diese Denkrichtungen ausweist, ist ihre mehr oder minder radikale Ablehnung der traditionellen Darstellungen und in der Folge die Revision der darauf basierenden Geschichtsauffassung. Darüber hinaus gibt es schließlich Ansätze, welche zwischen den ersten beiden pendeln beziehungsweise die ,integrative“ Modelle bevorzugen und dabei eine Synthese mit weitgehender Vereinbarkeit der einzelnen Quellen sehen. Die Bezeichnungen ,traditionell' und ,revisionistisch' sind nicht wertend zu verstehen. Der Autor ist sich bewusst, dass die Begriffe vereinfachend sind. Sie sollen lediglich dazu dienen, bestehende Unterschiede sichtbar zu machen und zusammenzufassen.

In der westlichen Islamwissenschaft wurde beim Versuch, die historische Entwicklung des Islam nachzuzeichnen, reichlich Gebrauch von islamischen Quellen gemacht. Sie wurden kritisch durchleuchtet mit dem Ziel, Geschichtlichkeit von Legende beziehungsweise Hagiographie zu trennen. Dieser ,klassische Ansatz' wurde zum Beispiel durch William Montgomery Watt in seinen Werken Muhammad. Prophet and Statesman und Islam. A Short History aus den Jahren 1961 und 1999 sowie 1957 von Rudi Paret in seinem Buch Mohammed und der Koran prominent vertreten.

Die revisionistischen Ansätze hingegen lehnen die islamischen Prophetenbiographien und Erzählungen zu historischen Ereignissen gänzlich als ,heilsgeschichtliches' Konstrukt ab. Das heißt, so meint man, hier werde im Grunde keinerlei Geschichte verarbeitet, sondern nur eine Heils- und Begründungsgeschichte des Islam geschrieben, welche durch und durch konstruiert sei. John Wansbroughs Buch Quranic Studies (1977) hat den revisionistischen Stein in vielerlei Hinsicht ins Rollen gebracht. Wansbrough argumentiert - weitgehend anhand literarischer Oberflächenmerkmale (Sinai 2009) -, der Koran sei nach und nach entstanden und habe seine endgültige inhaltliche Form wohl erst nach dem Jahr 800 und zwar in Mesopotamien erhalten. Auch Patricia Crone schloss sich in Hagarism. The Making of the Islamic World (1977) gemeinsam mit Michael Cook diesem Szenario an. Ihr zufolge fanden die expandierenden arabischen Stämme ein sektiererisches Milieu vor, aus welchem sie dann ihre eigene ,Religion“ konstruierten, deren Ursprung aus politischen Gründen in den ,polytheistischen' Hijâz verlegt wurde. Wesentlich für Wansbroughs Argumentation ist die von ihm angenommene enge Verflechtung von Koran, tafsîr- (exegetischen Werken) und sîra-Literatur (Prophetenbiographien). Crone versucht ganz ohne traditionelle islamische, vielmehr nur mit außerislamischen Quellen eine Rekonstruktion der frühislamischen Geschichte.

Einen Schritt weiter geht Jehuda Nevo in Crossroads to Islam (2003), welches nach seinem Tode von Judith Koren herausgegeben wurde. Hier wird nicht einmal mehr von arabischen Eroberungen ausgegangen, sondern von einem gezielten Rückzug der Byzantiner, deren Gebiet dann von sektiererischen Arabern übernommen wurde. Nevo lehnt die innerislamischen literarischen Quellen ebenfalls als völlig nutzlos und unglaubwürdig ab und stützt sich auf frühislamische Inschriften im Negev und auf Münzlegenden. Im deutschen Raum führt eine Gruppe um den Saarbrücker Emeritus für Religionswissenschaft Karl-Heinz Ohlig und den Numismatiker Volker Popp die Thesen von Nevo weiter (Ohlig/Puin 2005). 622 bis 627, nach dem Sieg des byzantinischen Kaisers Herakleius gegen die persischen Sassaniden, soll der kurz zuvor von letzteren besetzte syrisch-palästinische Raum von den Byzantinern aufgegeben und von zum Teil dort bereits ansässigen arabischen Stämmen in Besitz genommen worden sein. Als Abgrenzung zum byzantinischen Reich hätten sie ein nicht-trinitäres Christentum übernommen, welches dann zum ,Islam` ausgearbeitet wurde. Offenbar verorten die Autoren den Ursprung des Islam mittlerweile in der Gegend um Marw im Ostiran. Das Gesamtbild bleibt - zumindest für den Autor dieses Beitrags - unklar. Einen Propheten ,Muhammad' hat es nach diesem Ansatz nicht gegeben; dieser These hat sich neuerdings auch der Islamwissenschaftler Muhammad Sven Kalisch angeschlossen; für eine andere Sichtweise, die für eine frühere, ,traditionellere' Datierung der Entstehung des Islam eintritt, lohnt sich ein Blick in Fred Donners The early Islamic conquests (1981). 


\section{Diskussion}

\section{A. Einleitung}

Leider kann hier nur recht kursorisch auf die Diskussion eingegangen werden. Man beachte die Literaturhinweise am Ende.

Wansbroughs Verdienst ist es fraglos, einen allzu unbekümmerten Umgang mit islamischen Quellen zu problematisieren. Richtig ist sicher die grundsätzliche Feststellung, dass es sich bei der Sîra, der Prophetenbiographie, nicht primär um eine Chronik, sondern um ein soziopolitisches Werk handelt, welches auch auf Fragen des 8. respektive 9. Jahrhunderts antwortet und neue Elemente (etwa die Isrâ îliyyât, Erzählungen aus der hebräischen Bibel) aufnimmt. Die Sîra enthält gewiss legendäre Bestandteile. Trotzdem ist Wansbroughs Argumentation oft zirkulär (siehe Versteegh 1993; Sinai 2009: 25); der Text des Koran passt sehr wohl in ein ,klassischeres' Szenario (dazu unten mehr). Das gleiche - vor allem der Vorwurf der Zirkularität - gilt auch für Nevo (siehe Foss 2005; Johns 2003; Sinai 2009: 27). Ohligs und Popps Zweifel an der Existenz eines Propheten ,Muhammad' hat kaum Anhänger, die Lokalisierung von arabischen foederati (Hilfstruppen) im Marw hat kaum eine Basis.

\section{B. Religiöse Pluralität im Hijâz.}

Der Hijâz als Ausgangsort des Islam ist keineswegs unwahrscheinlich. Er stellt sich heute nicht als tabula rasa dar, sondern als Ort, an dem jüdische, christliche und heidnische Elemente aufeinandertrafen; unter ,heidnisch' sind hier die verschiedensten Richtungen zu verstehen wie unabhängige Mono- oder doch zumindest Henotheisten, Polytheisten und andere. Jedenfalls war die Religionszusammensetzung heterogen und nicht im Sinne einer organisierten Religion festgelegt. Unter anderem im Rahmen des Projektes „Corpus Coranicum" an der Berlin-Brandenburgischen Akademie der Wissenschaften (corpuscoranicum.de) konnte festgestellt werden, dass im Koran Begebenheiten reflektiert werden, und zwar nicht nur solche aus dem Norden und ein wenig aus dem Osten, sondern auch aus dem Süden der arabischen Halbinsel. Diese Reflektionen weisen recht zuverlässig auf eine Entstehung des Islam in der Mitte der arabischen Halbinsel hin. So wird etwa das Wort rahmân nicht nur, wie die Tradition es sieht, als Beschreibung Gottes mit ,der Barmherzige' benutzt, sondern quasi als eigenständiger Name neben Allâh. Im Koran heißt es: „Sagt bei der Anrufung ,Allâh“ oder ,Rahmân', was ihr auch sagt, Er hat die schönsten Namen“ (Sure 17:110, Übersetzung des Autors). Gleichzeitig gibt es etliche Suren, in den ausschließlich rahmân als Bezeichnung für den monotheistischen Gott gebraucht wird. Der Gottesname rahmân findet sich nun zugleich in nördlicher jüdischer Literatur, sowie vor allem auch im Jemen (Robin 2004). Auch die Prophetenrivalen Musaylima (Maslama) und alAswad beispielsweise predigten den rahmân. Allâh hingegen ist wiederum genuin arabischer Herkunft, allerdings mit Anklängen an das Syrische allâhâ (Kiltz 2011).

Wenn im Koran gegen ,polytheistische' Inhalte argumentiert wird, dann mag das auch zum Teil auf frühere Praktiken weisen und zum Teil auf andere monotheistische Gruppen zielen wie etwa trinitäre Christen (Hawting 1999). Das heißt, die Ermahnungen könne sich nicht nur auf tatsächlich noch lebendige, heidnische' Kulte beziehen, sondern auf mehr oder weniger ,monotheistische' Gruppen, denen vorgeworfen wird, sie setzten - zum Teil in anderer Form - alte, ,polytheistoide' Traditionen fort. Trotzdem sind gewisse ,heidnische' Elemente für Mekka anzunehmen (Saleh 2009). Im Koran werden mushrikûn (Menschen, die Gott etwas beigesellen, folglich seine einzige und alleinige Stellung verwässern oder leugnen, also ,Heiden' oder vielleicht ,nicht ausreichend monotheistische' Gruppen) direkt angesprochen. Unter anderem werden sie gemahnt, die Wunder des Einen Gottes anzuerkennen. So heißt es in Sure 16, Vers 11: „Damit [mit dem Regen] lässt Er für euch Korn sprießen und den Ölbaum und die Dattelpalme und die Trauben und Früchte aller Art. Wahrlich, darin liegt ein Zeichen für nachdenkende Leute.“ Crone wendet nun ein, dass angenommene Polytheisten 
und andere in Mekka nicht als Kultivatoren von Oliven, Weizen und Datteln in Frage kämen, da man dort zwar Viehzucht betrieben habe, aber keine Landwirtschaft. Es sei daher zweifelhaft, ob dieser Offenbarungsdiskurs tatsächlich in Mekka oder nicht doch an einem anderen Ort stattgefunden habe. So würden Oliven beispielsweise in Palästina angebaut, nicht aber auf der arabischen Halbinsel. Dieser Einwand ist aus Sicht des Projekts „Corpus Coranicum“ nicht stichhaltig. Zum einen waren am Handelsplatz Mekka sicherlich Leute aus Gegenden anwesend, an denen die genannten Erzeugnisse wuchsen. Noch wichtiger ist aber zum anderen, dass es sich um symbolträchtige Produkte handelt, die gewiss begehrt waren und eingehandelt wurden. Somit nennt der Koran in einem universalistischen Ton exquisite Erzeugnisse, an denen sich der ,Mensch' labt; mit ,euch' (lakum) sind wohl auch ,Leute' allgemein gemeint. Im folgenden Vers 14 wird von der Dienstbarmachung des Meeres gesprochen. Das muss ebenso wenig implizieren, dass alle Mekkaner Seefahrer gewesen sind oder der Ort des Offenbarungsdiskurses an eine Hafenstadt verlegt werden muss.

Mekka liegt von Orten umgeben, die hinsichtlich der Religion (mit starkem Monotheismus), der Kultur und der dort vorhandenen Erzeugnisse vielfältig und divers geprägt sind. Südlich, im Jemen finden wir Reiche mit zum Judentum konvertierten Herrschern (etwa Dhû Nuwâs) und Eroberungen christlicher Äthiopier. Die nördlich lebenden arabischen Stämme waren zum Teil schon früh zum Christentum verschiedener Prägung übergegangen. Vorislamische Dichtung, so zum Beispiel die des aus Tâ'if, einer nur eine Tagesreise von Mekka entfernt liegenden blühenden Oase, stammenden Dichters Umayya Ibn Abî al-Salt beinhaltet ebenfalls biblische Motive (Seidensticker 1996: 412). Der Osten der arabischen Halbinsel war persisch besetzt. Nicht zuletzt sei bemerkt, dass auch islamische Quellen von Konversionen zum Christentum in Mekka und Medina beziehungsweise (Handels-)Kontakten mit Christen kurz vor dem Islam sprechen (Osman 2005). Mekka hatte also Handelsbeziehungen, die Anknüpfungspunkte zu einer Vielzahl von Menschen und Ideen beinhalteten, sowie typischerweise eine relativ große religiöse Pluralitätstoleranz. Es spricht also alles dafür, dass die spätantiken religiösen Hauptdiskurse durchaus auch in Mekka zu hören waren.

\section{Die Situation des Propheten}

Da nun Muhammad Prophet einer weiteren abrahamischen Religion ist, ist auch der Begriff ummî (Sure 7, Vers 157 und 158: nabî wa-rasîl ummî) als Bezeichnung des Propheten verständlich. Die in der islamischen Tradition vorherrschende (jedoch keineswegs einzige) Interpretation als ,illiterat" ist schon rein sprachlich kaum $\mathrm{zu}$ halten. Im obigen Zusammenhang ist vielmehr eine Bedeutung ,Prophet der Völker, hebräisch gôyîm, syrisch 'ammê vorzuziehen. Muhammad ist demnach also ein Prophet für ein Volk, welches vorher noch keine ,Schrift' erhalten hat.

Die Vorstellung der Einteilung in Buchreligionen versus ,Heiden“ ist in der jüdischen und christlichen Spätantike üblich. Der Koran als kitâb ,Schrift' in 'arabî mubîn, in ,klarem Arabisch', zeigt die massive Relevanz der abrahamischen Buchreligionen, aber auch, dass die Araber sich noch nicht alle diesen zugehörig fühlten beziehungsweise offen für eine eigene, ,arabische' Version waren.

\section{Münzen}

Nicht unerwähnt bleiben, soll ferner die Rolle von Münzen in der ,revisionistischen“ Diskussion. Die frühen Münzen aus islamischer Zeit weisen noch knapp bis ans Ende des 7. Jahrhunderts christliche, im Osten sassanidisch/zoroastrische Symboliken auf, und erst in den 680er Jahren finden sich Aufschriften wie „Muhammad“ und „der Gesandte Gottes“ im Osten des islamischen Machtbereichs. Nevo und dann Ohlig und Popp wollen hierin eine Bestätigung ihrer These sehen, wonach es sich beim frühen Islam gar nicht um eine neue Religion, sondern um eine christliche Häresie gehandelt habe. Das Argument der christlichen Häresie wird allerdings schon allein dadurch unwahrscheinlich, dass die bereits bestehenden 
Münzen - nämlich byzantinische und sassanidische - einfach bloß weiter im Umlauf geblieben sin. Zumindest die Münzen im Osten mit eindeutig zoroastrischer Symbolik (etwa dem Feueraltar) wären damit nicht zu erklären. Tatsächlich ist der Befund gar nichts Ungewöhnliches. Wie zum Beispiel Stefan Heidemann (2007, 2009) darlegt, sind Münzen konservativ. Eine Münzumstellung braucht - zumal in einer vorindustriellen Gesellschaft schlicht Zeit. Die schrittweise Übergang von sassanidischer beziehungsweise byzantinischer Symbolik zu einer gänzlich islamisch geprägten lässt sich anhand der Münzfunde gut nachvollziehen ohne, dass hier von einer späteren ,Konstruktion“ einer neuen Religion ausgegangen werden muss. Als Beispiel für einen ähnlich gelagerten Fall sei hier der Übergang von der Seleukidenherrschaft zu der der iranischen Arsakiden genannt. Die Seleukiden sind Nachfolger von Alexander dem Großen und bedienten sich auf ihren Münzen der griechischen und hellenistischen Symbolik. Die Arsakiden berufen sich wieder auf rein iranische Traditionen. Hier findet also ein religiöser und dynastischer Umbruch statt. Das Griechische ist als Herrschaftssprache nicht mehr in Gebrauch, ja es ist sogar - zumindest im Osten - die Sprache des größten Widersachers der Arsakiden, nämlich der Römer. Trotzdem sind Münzen mit griechischer Aufschrift noch über 200 Jahre lang in Gebrauch. Verglichen damit ist die ,Umstellung' in der islamischen Welt sogar als sehr zügig zu bezeichnen (circa 70 Jahre). Die Beispiele ließen sich beliebig vermehren. Ein administrativer Normalfall, wie es die Anpassung von Münzen an neue politische und religiöse Gegebenheiten ist, soll hier also als Kronzeuge für ,falsche Geschichtsschreibung' herhalten.

\section{E. Außerislamische literarische Quellen}

Eine sehr wichtige Rolle spielen natürlich auch die außerislamischen literarischen Quellen. Doch gerade diese Quellen bestärken die traditionelle Datierung und auch die Existenz eines Propheten Muhammad. So fasst Patricia Crone in einem 2008 erschienenen Artikel zusammen: „Es gibt keinen Zweifel, dass Mohammad existiert hat, trotz gelegentlicher Versuche dies zu leugnen“. Paraphrasiert führt sie weiterhin aus: Frühe, außerislamische literarische Quellen berichten über Muhammad. Bereits um etwa 632 bis 634 beschreibt die Doctrina Jacobi, ein ,falscher Prophet ist unter den Sarazenen aufgetreten“. Die Doctrina weist ihn freilich als einen Scharlatan zurück, denn Propheten kämen nicht „mit Schwert und Streitwagen“ (Déroche 1991: 57). Möglicherweise haben wir hier einen noch zeitgenössischen Bericht über Muhammad. Muhammads Tod wird traditionell gregorianisch auf das Jahr 632 datiert. Es gibt jedoch muslimische Quellen, welche den Anfang der islamischen Zeitrechnung nicht auf 622, sondern auf 624 bis 625 setzen. Damit fiele der griechische Bericht in die letzten Lebensjahre des Propheten.

Der Hinweis auf die kriegerische Tätigkeit deckt sich mit islamischen Schriften. Eine armenische Quelle aus der zweiten Hälfte des 7. Jahrhunderts identifiziert Muhammad namentlich und beschreibt erkennbar seine Lehren. Ergänzend sei hier Thomas der Presbyter (Mitte des 7. Jahrhunderts) genannt, der ebenfalls Eroberungen durch die „[Araber von] Muhmd“ im Jahre 635 bis 636 beschreibt; ,die Araber von“, syrisch tayyâyê $d$-, ist eine Ergänzung von Hoyland/Palmer (1993). Die Lesung MWHMD ist jedoch sicher. Der arabische Name Muhammad muss im Syrischen so geschrieben werden, eine andere Lesung ist nicht möglich.

Es gibt allerdings auch hier Details, die sich nicht eins zu eins mit der islamischen Tradition decken. So verkündet nach der Doctrina Jacobi der Anführer der Araber den Messias und hat eine jüdische Gefolgschaft. Nun sind auch die außerislamischen Quellen, ebenso wie die innerislamischen immer vor dem Hintergrund ihrer politisch-religiösen Stoßrichtung zu sehen. Im vorliegenden Fall handelt es sich um eine antijüdische Schrift in der Jakob, ein Jude, zum Christentum übergetreten ist. Die generell freundliche Aufnahme der arabischen Eroberung durch die jüdischen Bewohner Palästinas - Palästina wird hier im Sinne der 
römischen beziehungsweise später byzantinischen Provinz gebraucht - mag hier mitschwingen.

\section{F. Integrativer Ansatz}

Der dritte, der, integrative' Ansatz sieht die Herkunft des Propheten Muhammad aus Mekka als wahrscheinlich an und versucht, nach Meinung des Autors erfolgreich, die verschiedenen Quellen zu harmonisieren. Dabei hilft nach den Worten Angelika Neuwirths „nur die Aufarbeitung des bestehenden Forschungsdefizits: es gilt ,von außen', historisch, das kulturelle und religiöse Umfeld [des Korans] in seiner ganzen Komplexität sichtbar zu machen. Es gilt aber auch, den Gegenweg einzuschlagen und den Koran selbst - zwar nicht als historisch unmittelbar auswertbare Quelle, aber doch - als literarisch-kodierte Aussage über seine Zeit und über seine Genese ernst zu nehmen.“ (2004:132) Was kann also der Text, der sicher als Diskurs innerhalb einer Gemeinde in statu nascendi und später dann in statu affirmandi entstand, über die Zeit, die Umstände und den Ort seiner Entstehung sagen? Hierbei sind als äußere Quellen nicht nur die historischen Werke und Realien heranzuziehen. Es ist vielmehr ebenso notwendig, die geistige Umwelt des Koran zu rekonstruieren. Verschiedene Ansätze finden sich z.B. bei Gabriel Said Reynolds (2008), Michael Cuypers (2009) und Neuwirth, Sinai, Marx (2009). Ein noch recht junges Projekt ist das „Corpus Coranicum" (Marx 2008), welches unter Anderem sogenannte Intertexte untersucht.. Dabei handelt es sich etwa um christliche und jüdische religiöse Abfassungen aus der zeitlichen und geographischen Umwelt des Koran. Diese Texte finden im Koran Anklang und werden in ihm diskursiv reflektiert. Das heißt, es sind Texte, die bekannt waren und als relevant im Koran aufgearbeitet und gedeutet werden. Demzufolge wurden die Strömungen nicht ,kopiert` oder passiv aufgenommen, wie das in früheren Werken oft angenommen wird. Der Koran behandelt Themen und nimmt Stellung; ein Beispiel für eine integrative Haltung in Bezug auf die Sîra, also die Prophetenbiographie, ist Andreas Görkes und Gregor Schoelers Die ältesten Berichte über das Leben Muhammads (2008).

\section{Ausblick}

Neben der Aufarbeitung der bereits genannten Quellen würden wohl auch archäologische Ausgrabungen auf dem Gebiet der arabischen Halbinsel reichen Erkenntniszugewinn bescheren. Von dieser Grundlage aus, einem literarisch-linguistischem und - soweit möglich - archäologischen Panoptikum, kann dann auch der weitere Verlauf der Geschichte betrachtet werden. Es ist das Verdienst der revisionistischen Richtung auf Probleme in der traditionellen Geschichtsschreibung hinzuweisen. Manchmal scheint es allerdings, als sei hier das Kind mit dem Bade ausgeschüttet worden. Oftmals wird obscurum per obscurius erklärt, so etwa im Falle der angenommenen Entstehung des Koran im Marw. Auch Wansbroughs oder Nevos Annahmen passen nicht nur nicht zu der islamischen Überlieferung, sondern ebenso wenig zur zeitgenössischen außerislamischen Literatur. Um zu einem kohärenteren Bild zu kommen, sollte die islamische Überlieferung kritisch integriert werden, statt sie komplett auszublenden (siehe auch Neuwirth 2004: 132). Es gilt, alle Quellen, aber auch alle Probleme der traditionellen Überlieferung mit kritischem Abstand ernst zu nehmen - so wie das von der überwältigenden Mehrheit der Forscher auch betrieben wird. Wie die obigen Ausführungen zeigen, hat die Islamwissenschaft in den letzten Jahren eine deutliche Belebung erfahren. Letzten Endes kann ein dialektischer Prozess des Austauschs zwischen den verschiedenen Denkrichtungen in Bezug auf die frühislamische Geschichte - wie er mittlerweile auch schon stattfindet - nur Gewinn bringen.

\section{Literaturliste}

Burgmer, C. (Hrsg.) (2004): Streit um den Koran. Die Luxenberg-Debatte. Standpunkte und Hintergründe. Berlin. 
Crone, P. (2004): Meccan trade and the rise of Islam. Piscataway, N.J.

Crone, P. (2008): „What do we actually know about Mohammed? “, in: opendemocracy.net.

Crone, P. (2008a): From Arabian tribes to Islamic empire. Army, state and society in the Near East c.600-850. Aldershot u.a.

Crone, P. und M. Cook (1977): Hagarism. The making of the Islamic world. Cambridge u.a.

Cuypers, M. (2009): The banquet. A reading of the fifth sura of the Qur'an. Miami, Fl.

Déroche, V. (Hrsg./Übers.) (1991): „Doctrina Jacobi nuper Baptizati“, in: G. Dagron und V. Déroche: „Juifs et chrétiens dans l'Orient du VIIe siècle“, in: Travaux et mémoires 11(1991), S. 17-248.

Donner, F. M. (1981): The early Islamic conquests. Princeton, N.J.

Foss, C. (2005): „An Unorthodox View of the Rise of Islam“, in: Journal of Roman Archaeology 18(2005), S. 771-774.

Görke, A. und G. Schoeler (2008): Die ältesten Berichte über das Leben Muhammads. Das Korpus 'Urwa Ibn az-Zubair. Princeton, N.J.

Hawting, G. R. (1999): The idea of idolatry and the emergence of Islam. From polemic to history. Cambridge u.a.

Heidemann, S. (2007): „Münzen sind konservativ. Der frühe Islam im Spiegel des numismatischen Befundes“, in: $F A Z, 28.2 .07$ [uni-jena.de].

Heidemann, S. (2009): ,The development of the representation of the early Islamic empire and its religion on coin imagery", in: A. Neuwirth, N. Sinai und M. Marx (Hrsg.): The Qur'an in context. Literary and historical investigations into the Qur 'anic milieu. Leiden, S. 149-195.

Hinds, M. u.a. (1996): Studies in early Islamic history. Princeton, N.J.

Hoyland, R. G. (1997): Seeing Islam as others saw it. A survey and evaluation of Christian, Jewish, and Zoroastrian writings on early Islam. Princeton, N.J.

Johns, J. (2003): „Archaeology and the history of early Islam. The first seventy years“, Journal of the Economic and Social History of the Orient 46(2003), S. 411-436.

Kiltz, D. [2011]: „The relationship between Quranic Allâh and Syriac Allâhâ.” [im Druck; Beitrag für den geplanten Sammelband zur Internationalen Tagung „Christen im Orient Geschichte und Bedeutung" vom 28. bis 29. November 2008 in Berlin].

Marx, M. (2008): "Ein Koran-Forschungsprojekt in der Tradition

der Wissenschaft des Judentums: Zur Programmatik des Akademienvorhabens

Corpus Coranicum”. In: Dirk Hartwig u. a. (HG.), Im vollen Licht der

Geschichte. Die Wissenschaft des Judentums und die Anfänge der

kritischen Koranforschung. Würzburg, S. 41-54.

Neuwirth, A. (2004): „Zur Archäologie einer Heiligen Schrift- Überlegungen zum Koran vor seiner Kompilation“, in: C. Burgmer (Hrsg.): Streit um den Koran. Die LuxenbergDebatte. Standpunkte und Hintergründe. Berlin, S. 82-97.

Neuwirth, A. (2007): Studien zur Komposition der mekkanischen Suren. Die literarische Form des Koran - ein Zeugnis seiner Historizität. Berlin.

Neuwirth, A., Sinai, N. \& Marx, M. (2009) The Qur'an in Context:

Historical and Literary Investigations into the Qur'anic Milieu (Texts

and Studies on the Qur'an). Leiden.

Ohlig, K.H. und G.R. Puin (Hrsg.) (2005): Die dunklen Anfänge. Neue Forschungen zur Entstehung und frühen Geschichte des Islam. Berlin.

Osman, Gh. (2005): „Pre-Islamic Arab Converts to Christianity in Mecca and Medina: An Investigation into the Arabic Sources", in: The Muslim World 95 (2005), S. 67-80.

Palmer, A. / S.P. Brock und R. Hoyland (1993): Seventh century in the West-Syrian chronicles. Liverpool.

Reynolds, G.S. (2008): The Qur'ân in its historical context. London u.a. 
Robin, Chr.-J. (2004): „Himyar et Israël”. Comptes rendus des séances de l'année 2004 Avril-Juin.

Académie des inscriptions et belles-lettres 2, 831-908. Paris.

Saleh, W. (2009): „The etymological fallacy and Quranic studies. Muhammad, paradise, and late antiquity", A. Neuwirth, N. Sinai und M. Marx (Hrsg.): The Qur'an in context. Literary and historical investigations into the Qur' anic milieu. Leiden. [safarmer.com]

Seidensticker, T. (1996): „The authenticity of the poems ascribed to Umayya Ibn Abi al-Salt”, in. J.R. Smart (Hrsg.): Tradition and modernity in Arabic language and literature. Richmond, S. 87-101.

Sinai, N. (2009): Fortschreibung und Auslegung. Studien zur frühen Koraninterpretation. Wiesbaden.

Versteegh, C.H.M. (1993): Arabic grammar and Qur'ânic exegesis in early Islam. Leiden. 